Math. Z. 206, 501-504 (1991)

Wathematische

zeltschrift

(C) Springer-Verlag 1991

Erratum:

\title{
A precise result on the boundary regularity of biholomorphic mappings
}

\author{
László Lempert \\ Purdue University, West Lafayette, IN 47907, USA
}

Math. Z. 193, 559-579 (1986)

1. Mr. Ralf Kampmann has called my attention to two errors in the paper in question, [3]. Although in the meantime another paper has appeared which proves essentially the same regularity result for biholomorphic mappings as does [3] (see [1]), I would like to correct the mistakes here. One of the reasons is that [3] discusses regularity properties of the Kobayashi metric, too, and precise regularity of the Kobayashi metric may be of interest in other types of questions as well (cf. $[4,5]$ ).

The errors are in the proof of Theorem 4.1 on page 566 , line-5 in [3]. The first is a simple misprint: $\Phi$ should read $\Theta$. The second is that this mapping $\Theta$ is not Hölder continuous of order $C^{k-s}$. Therefore the implicit function theorem cannot be applied and does not yield the regularity of $\Phi$ claimed in Theorem 4.1. However, $\Theta$ is $C^{k-s}$ when restricted to a smaller subset of its domain of definition in [3], and, as we are going to show here, this is sufficient to correct the proof of Theorem 4.1.

2. First recall Whitney's notion of Hölder continuity of functions defined on not necessarily open sets. Let $A, E$ be Banach spaces, $\mathscr{A} \subset A$ a subset. A mapping $\phi: \mathscr{A} \rightarrow E$ is $C^{l}(0<l<1)$ if $\left\|\phi\left(a_{1}\right)-\phi\left(a_{2}\right)\right\|_{E} \leqq$ const $\left\|a_{1}-a_{2}\right\|_{A}^{l}$ for $a_{1}, a_{2} \in \mathscr{A} . \phi$ is called differentiable if for every $a \in \mathscr{A}$ there is a continuous linear mapping $L_{a} \in L(A, E)$ such that

$$
\left\|\phi\left(a_{1}\right)-\phi(a)-L_{a}\left(a_{1}-a\right)\right\|_{E}=o\left(\left\|a_{1}-a\right\|_{A}\right) \quad \text { as } \mathscr{A} \ni a_{1} \rightarrow a
$$

Finally, if $l>1$ is non integral then $\phi$ is (inductively) defined to be $C^{l}$ if it is differentiable and $L_{a}$ can be chosen so that the mapping $\mathscr{A} \ni a \mapsto L_{a} \in L(A, E)$ is $C^{l-1}$. We shall denote $L_{a}$ by $\partial \phi / \partial a$. Although $\partial \phi / \partial a$ is not uniquely determined, when $\phi$ is $C^{l}, \partial \phi / \partial a$ will always denote a $C^{l-1}$ Hölder continuous choice of the partial derivative. 
Let now $R>0$. With notation as in [3], let $\Xi_{m}(R)=\Xi_{m} \subset X_{m}$ denote the set of those mappings $f: \partial U \rightarrow \mathbb{C}^{m}$ whose components are in $C^{1}$ with $C^{1}$-norm less than $R$.

Proposition 1 Suppose $K: \mathbb{C}^{m} \rightarrow \mathbb{C}$ is a function of class $C^{l}, l>0,\{l\}>s$. Then the mapping $\widetilde{K}: \Xi_{m} \rightarrow X_{1}$ defined by $\widetilde{K}(g)=K \circ g$ is of class $C^{l-s}$.

Proof. Assume first $0<l<1$, and let $g, h \in \Xi_{m}$. For $x \in \partial U$

and for

$$
\begin{aligned}
|K(g(x))-K(h(x))| & \leqq\|K\|_{C^{l}}|g(x)-h(x)|^{l} \\
& \leqq\|K\|_{C^{l}}\|g-h\|_{X_{m}}^{l-s}
\end{aligned}
$$

$$
\Delta=(K(g(x))-K(h(x)))-(K(g(y)-K(h(y)))
$$

with $x, y \in \partial U$ we have the estimates

$$
\begin{aligned}
|\Delta| & \leqq\|K\|_{C^{\prime}}\left(|g(x)-h(x)|^{l}+|g(y)-h(y)|^{l}\right) \\
& \leqq 2\|K\|_{C^{\prime}}\|g-h\|_{X_{m}}^{l}, \\
|\Delta| & \leqq 2\|K\|_{C^{\prime}} R^{l}|x-y|^{l} .
\end{aligned}
$$

The two latter inequalities imply

$$
|A| \leqq \text { const }\|g-h\|_{X_{m}}^{l-s}|x-y|^{s} .
$$

This, together with (1) prove that $\tilde{K}$ is $C^{l}$.

Next, if $1<l<2$, write

$$
\begin{aligned}
K(g(x)+h(x))-K(g(x)) & =K^{\prime}(g(x)) h(x)+R(x), \\
R(x) & =\int_{0}^{1}\left(K^{\prime}(g(x)+t h(x))-K^{\prime}(g(x))\right) h(x) d t,
\end{aligned}
$$

where, this time, $g, g+h \in \Xi_{m}$. From what has already been proved it follows that

$$
\|R\|_{X_{1}}=o\left(\|h\|_{X_{m}}\right) \text { as } h \rightarrow 0 .
$$

Hence $\widetilde{K}$ is differentiable and its derivative in $g$ can be given by the map

$$
X_{m} \ni \gamma \mapsto\left(K^{\prime} \circ g\right) \gamma \in X_{1} .
$$

Thus, by the first step of the proof, $\widetilde{K} \in C^{l-s}$.

The proof for general $l$ is done by an obvious induction.

Next we check a simple version of the implicit function theorem. Let $A, B$, and $E$ be Banach spaces, $\mathscr{A} \in A$ a convex subset. 


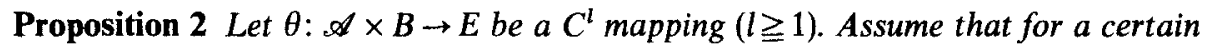
point $\left(a_{0}, b_{0}\right) \in \mathscr{A} \times B \theta\left(a_{0}, b_{0}\right)=0$ and that for an appropriate choice of the partial derivative $\partial \theta / \partial a$

$$
\frac{\partial \theta}{\partial a}\left(a_{0}, b_{0}\right): A \rightarrow E
$$

is an isomorphism.

If now $\varphi$ is a continuous mapping of some neighborhood $G$ of $b_{0} \in B$ into $\mathscr{A}$ such that $\varphi\left(b_{0}\right)=a_{0}$ and $\theta(\varphi(b), b) \equiv 0$, then $\varphi$ is of class $C^{l}$ near $b_{0}$.

Proof. For $b, b_{1} \in G$ write

$$
\begin{aligned}
0 & =\theta\left(\varphi\left(b_{1}\right), b_{1}\right)-\theta(\varphi(b), b) \\
& =\frac{\partial \theta}{\partial a}(\varphi(b), b)\left(\varphi\left(b_{1}\right)-\varphi(b)\right)+\frac{\partial \theta}{\partial b}(\varphi(b), b)\left(b_{1}-b\right)+R, \\
\|R\|_{E} & =o(1)\left(\left\|\varphi\left(b_{1}\right)-\varphi(b)\right\|_{A}+\left\|b_{1}-b\right\|_{B}\right) \quad\left(b_{1} \rightarrow b\right) .
\end{aligned}
$$

When $b$ is near $b_{0}, \partial \theta / \partial a(\varphi(b), b): A \rightarrow E$ is an isomorphism, and, indeed, the norm of the inverse mapping

$$
\left(\frac{\partial \theta}{\partial a}(\varphi(b), b)\right)^{-1}: E \rightarrow A
$$

is bounded. (2) and (3) now imply

and so by (3)

$$
\left\|\varphi\left(b_{1}\right)-\varphi(b)\right\|_{A}=O\left(\left\|b_{1}-b\right\|_{B}\right) \quad\left(b_{1} \rightarrow b\right),
$$

$$
\|R\|_{E}=o\left(\left\|b_{1}-b\right\|_{B}\right)
$$

Applying the mapping (4) to (2), we see that $\varphi$ is differentiable and

$$
\frac{\partial \varphi(b)}{\partial b}=-\left(\frac{\partial \theta}{\partial a}(\varphi(b), b)\right)^{-1} \frac{\partial \theta}{\partial b}(\varphi(b), b) .
$$

Using this formula a simple induction proves that $\varphi$ is $l$-Hölder continuous near $b_{0}$.

3. There is one more fact that we shall need. Let $D$ be as in Lemma 3.1 of [3], and $f: U \rightarrow D$ an extremal map. By the above lemma, $f \in C^{k}(\bar{U})$.

Proposition 3 If $f(0)$ belongs to a compact subset $\Sigma \subset D$ then $\|f\|_{C^{k}} \leqq c(\Sigma, D)$, where the constant $c(\Sigma, D)$ does not depend on $f$.

Proof. That the $C^{1 / 2}$ norm of $f$ is uniformly bounded follows from the a priori estimates of [2] when $k>6$ and the approximation argument explained on bottom of page 562 and top of the next page in [3], when $\partial D$ is just $C^{2}$. Having uniform $C^{1 / 2}$ estimates at hand, the proofs that $f \in C^{k}(\widetilde{U})$ also yield uniform $C^{k}$ estimates, as is easily checked.

Corollary. The mapping $\Phi$ in Theorem 4.1 in [3] is continuous. 
Proof. Suppose $w_{j}, w \in D, v_{j}, v \in \mathbb{C}^{n} \backslash\{0\}, w_{j} \rightarrow w, v_{j} \rightarrow v$, and the extremal mappings $f_{j}=\Phi\left(w_{j}, v_{j}\right)$ do not converge to $f=\Phi(w, v)$ in $Y_{n}$.

Since the $C^{k}$-norms of $f_{j}$ are bounded, by passing to a subsequence, we can assume $f_{j} \rightarrow g \neq f$ in the space $C^{s}$. But the limit of extremal mappings being extremal, $g(0)=w=f(0)$, and $g^{\prime}(0)=\lambda_{1} v, f^{\prime}(0)=\lambda_{2} v$, we obtain $g=f$, a contradiction.

After these preparations, in the proof of Theorem 4.1, the following modifications have to be made. If we want to prove that $\Phi$ is $C^{k-s}$ in a neighborhood of $(W, V) \in D \times \mathbb{C}^{n} \backslash\{0\}$, choose $R>0$ so that for any extremal mapping $f: U \rightarrow D$ with $f(0)$ near $W$ the $C^{1}$ norm of $f$ is less than $R$. Next, the mapping $\Theta$ should be defined only on a neighborhood of $(F, W, A, V)$ in

$$
\left(Y_{n} \cap \Xi_{m}(R)\right) \oplus \mathbb{C}^{n} \oplus \mathbb{R} \oplus \mathbb{C}^{n},
$$

instead of $Y_{n} \oplus \mathbb{C}^{n} \oplus \mathbb{R} \oplus \mathbb{C}^{n}$. It follows from Proposition 1 that $\Theta$ is $C^{k-s}$.

We want to apply Proposition 2. Let $A=Y_{n} \oplus \mathbb{R}, B=\mathbb{C}^{n} \oplus \mathbb{C}^{n}, E$ $=T \oplus Y_{n-1}^{\prime} \oplus \mathbb{C}^{n} \oplus \mathbb{C}^{n}, \mathscr{A}$ a neighborhood of $a_{0}=(F, A)$ in $Y_{n} \cap \Xi_{n}(R), b_{0}$ $=(W, V), \theta=\Theta$ and finally let $\varphi$ defined by

$$
\varphi(w, v)=(f, \lambda)
$$

where $f=\Phi(w, v)$ and $\lambda$ is such that $\lambda v=f^{\prime}(0)$.

By the corollary above $\varphi$ is continuous. The computations on pp 567-568 in [3] show that $\partial \theta / \partial a\left(a_{0}, b_{0}\right)=L$ is an isomorphism between $A$ and $E$. Hence Proposition 2 implies that $\varphi$, hence also $\Phi$, is $C^{k-s}$, as claimed.

\section{References}

1. Hasanov, S.V., Pinchuk, S.I.: Asymptotically holomorphic functions. Mat. Sb. 134(176), 546555 (1987)

2. Lempert, L.: La métrique de Kobayashi et la représentation des domaines sur la boule. Bull. Soc. Math. Fr. 109, 427-474 (1981)

3. Lempert, L.: A precise result on the boundary regularity of biholomorphic mappings. Math. Z. 193, 559-579 (1986)

4. Lempert, L.: Complex geometry in convex domains. Proceedings of the International Congress of Mathematicians. Berkeley, California: 1986

5. Lempert, L.: Holomorphic invariants, normal forms, and the moduli space of convex domains. Ann. Math., II. Ser. 128, 43-78 (1988) 